\title{
5. Dielectric Constants and Absorption Indices of Several Alcohols for Short Electric Waves."
}

\author{
By San-ichiro Mizushima. \\ Chemical Institute, Faculty of Science, Tokyo Imperial University.
}

(Comm. by S. Nakamura, M.I.A., Jan. 12, 1929.)

Dielectric constants $\varepsilon$ and absorption indices $x$ of several alcohols were measured for the wave-length of about $60 \mathrm{~cm}$. as the continuation of the previous work. ${ }^{2)}$ The results are shown in Table 1, in which some of the former results ${ }^{3)}$ measured for longer wave-lengths are also included in order to show the anomalous dispersion.

Table 1.

\begin{tabular}{|c|c|c|c|c|c|c|c|c|c|}
\hline Substance & $\begin{array}{l}\text { Ten } \\
\text { length in }\end{array}$ & era- & $60^{\circ}$ & $40^{\circ}$ & $20^{\circ}$ & $0^{\circ}$ & $-20^{\circ}$ & $-40^{\circ}$ & $-60^{\circ}$ \\
\hline $\mathrm{CH}_{3} \mathrm{OH}$ & $\begin{array}{r}5000 \\
950 \\
308 \\
58.0\end{array}$ & $\begin{array}{l}\varepsilon \\
\varepsilon \\
\varepsilon \\
\varepsilon \\
\chi\end{array}$ & $\begin{array}{l}- \\
- \\
- \\
-\end{array}$ & $\begin{array}{l}29 \\
31 \\
29.5 \\
28.3 \\
0.05\end{array}$ & $\begin{array}{l}32 \\
34 \\
33 \\
29.8 \\
0.08\end{array}$ & $\begin{array}{l}35 \\
37 \\
36.5 \\
29.2 \\
0.12\end{array}$ & $\begin{array}{c}39 \\
40 \\
40 \\
25.8 \\
0.19\end{array}$ & $\begin{array}{c}43 \\
45 \\
43 \\
20.2 \\
0.26\end{array}$ & $\begin{array}{l}47.5 \\
51 \\
\overline{13.2} \\
0.34\end{array}$ \\
\hline $\mathrm{C}_{3} \mathrm{H}_{7} \mathrm{OH}$ & $\begin{array}{r}5000 \\
950 \\
308 \\
57.8\end{array}$ & $\begin{array}{l}\varepsilon \\
\varepsilon \\
\varepsilon \\
\varepsilon \\
\gamma\end{array}$ & $\begin{array}{l}- \\
16 \\
17 \\
13.5 \\
0.14\end{array}$ & $\begin{array}{l}18.5 \\
19 \\
19 \\
11.4 \\
0.21\end{array}$ & $\begin{array}{l}21.5 \\
22 \\
20.5 \\
7.7 \\
0.29\end{array}$ & $\begin{array}{l}24 \\
25 \\
17.5 \\
3.9 \\
0.37\end{array}$ & $\begin{array}{l}27.5 \\
24 \\
11 \\
3.1 \\
0.30\end{array}$ & $\begin{array}{l}30 \\
15 \\
6.5 \\
3.1 \\
0.19\end{array}$ & $\begin{array}{l}24 \\
7 \\
5 \\
3.0 \\
0.12\end{array}$ \\
\hline $\mathrm{iC}_{4} \mathrm{H}_{9} \mathrm{OH}$ & $\begin{array}{r}5000 \\
950 \\
308 \\
57.8\end{array}$ & $\begin{array}{l}\varepsilon \\
\varepsilon \\
\varepsilon \\
\varepsilon \\
x\end{array}$ & $\begin{array}{l}\overline{14} \\
14 \\
11.9 \\
0.16\end{array}$ & $\begin{array}{l}17 \\
16 \\
16 \\
8.6 \\
0.24\end{array}$ & $\begin{array}{l}19.5 \\
19 \\
16 \\
4.9 \\
0.32\end{array}$ & $\begin{array}{l}22 \\
20 \\
10 \\
2.8 \\
0.33\end{array}$ & $\begin{array}{l}24 \\
11 \\
4.5 \\
2.8 \\
0.18\end{array}$ & $\begin{array}{c}18.5 \\
5 \\
3.5 \\
2.8 \\
0.08\end{array}$ & $\begin{array}{r}6 \\
4 \\
3 \\
- \\
-\end{array}$ \\
\hline $\mathrm{C}_{5} \mathrm{H}_{11} \mathrm{OH}$ & $\begin{array}{r}5000 \\
950 \\
308 \\
57.8\end{array}$ & $\begin{array}{l}\varepsilon \\
\varepsilon \\
\varepsilon \\
\varepsilon \\
x\end{array}$ & $\begin{array}{l}\overline{11} \\
12 \\
9.3 \\
0.15\end{array}$ & $\begin{array}{l}-\overline{13} \\
13.5 \\
5.7 \\
0.23\end{array}$ & $\begin{array}{l}15 \\
16 \\
13 \\
2.8 \\
0.32\end{array}$ & $\begin{array}{l}17.5 \\
17 \\
7.5 \\
2.7 \\
0.23\end{array}$ & $\begin{array}{l}19 \\
10 \\
4 \\
2.6 \\
0.11\end{array}$ & $\begin{array}{l}13 \\
5 \\
3 \\
2.6 \\
0.06\end{array}$ & $\begin{array}{l}5.5 \\
4 \\
3 \\
2.6 \\
0.04\end{array}$ \\
\hline $\mathrm{C}_{3} \mathrm{H}_{5}(\mathrm{OH})_{3}$ & $\begin{array}{c}5000 \\
950 \\
308 \\
57.8\end{array}$ & $\begin{array}{l}\varepsilon \\
\varepsilon \\
\varepsilon \\
\varepsilon \\
\chi\end{array}$ & $\begin{array}{c}\overline{37} \\
\overline{24.0} \\
0.22\end{array}$ & $\begin{array}{l}39 \\
40 \\
40.5 \\
13.6 \\
0.32\end{array}$ & $\begin{array}{l}43 \\
41 \\
28.5 \\
6.0 \\
0.32\end{array}$ & $\begin{array}{l}36 \\
20 \\
9 \\
3.1 \\
0.21\end{array}$ & $\begin{array}{l}13 \\
8 \\
5 \\
3.0 \\
0.10\end{array}$ & $\begin{array}{l}6 \\
4 \\
4 \\
3.0 \\
0.03\end{array}$ & $\begin{array}{l}4.5 \\
4 \\
3 \\
- \\
-\end{array}$ \\
\hline
\end{tabular}

1) Details are published in the Scientific Papers I.P.C.R., 9 (1928), 209.

2) Mizushima, Proc., 4 (1928), 205.

3) Mizushima, Phys. Zeit., 28 (1927), 418: Bull. Chem. Soc. Japan, 1 (1926), 47, $83,115,143$ and 163. 
As shown in the table, the region of anomalous dispersion is shifted towards longer wave-lengths when the temperature is lowered, as well as when a molecule becomes more complicated for a homologous series. This agrees with Debye's dipole theory on anomalous dispersion. ${ }^{1)}$ If we calculate the molecular radius of each substance by use of his formula, we find a constant value which is almost coincident with that obtained by other methods, although the experimental data extend over a wide range of temperature and wave-length. (See Table 2.) The results with glycerine, however, are not in accord with the dipole theory. The discrepancy would be accounted for, if glycerine be colloidally dispersed so that the effective viscosity acting against the rotation of molecule is quite different from the viscosity of the liquid in bulk. The superiority of the dipole theory to the classical electron theory was alreay pointed out in the previous paper.

For the sake of comparison static dielectric constant $\varepsilon_{0}$ and optic dielectric constant $\varepsilon_{\infty}$ (square of refractive index for D-line) of these alcohols are given in Table 3 .

The writer expresses his best thanks to Prof. M. Katayama for his kind guidance and to Mr. Onishi for his assistance in the experiment.

Table 2. Molecular Radii.

\begin{tabular}{|c|c|c|}
\hline Substance $\quad$ Method & $\begin{array}{l}\text { Critical temperature and } \\
\text { critical pressure }\end{array}$ & The present experiment \\
\hline $\mathrm{CH}_{3} \mathrm{OH}$ & $1.9 \times 10^{-8} \mathrm{~cm}$ & $1.7 \times 10^{-8} \mathrm{~cm}$ \\
\hline $\mathrm{C}_{3} \mathrm{H}_{7} \mathrm{OH}$ & $2.2 \quad$, & $2.3 \quad, "$ \\
\hline $\mathrm{iC}_{4} \mathrm{H}_{9} \mathrm{OH}$ & $2.2 \quad "$, & $2.3 \quad "$, \\
\hline $\mathrm{C}_{5} \mathrm{H}_{11} \mathrm{OH}$ & & 2.5 \\
\hline
\end{tabular}

Table 3.

\begin{tabular}{c|c|c|c|c|c|c|c|c}
\hline & & $60^{\circ}$ & $40^{\circ}$ & $20^{\circ}$ & $0^{\circ}$ & $-20^{\circ}$ & $-40^{\circ}$ & $-60^{\circ}$ \\
\hline \multirow{2}{*}{$\mathrm{CH}_{3} \mathrm{OH}$} & $\varepsilon_{0}$ & - & 29.0 & 31.5 & 35.2 & 39.0 & 43.5 & 48.0 \\
& $\varepsilon_{\infty}$ & - & - & - & 1.80 & 1.82 & 1.85 & 1.88 \\
\hline $\mathrm{C}_{3} \mathrm{H}_{7} \mathrm{OH}$ & $\varepsilon_{0}$ & 16.5 & 19.3 & 22.2 & 24.8 & 27.5 & 30.5 & 33.7 \\
& $\varepsilon_{\infty}$ & 1.86 & 1.89 & 1.92 & 1.95 & 1.98 & 2.02 & 2.05 \\
\hline \multirow{2}{*}{$\mathrm{iC}_{4} \mathrm{H}_{9} \mathrm{OH}$} & $\varepsilon_{0}$ & 15.0 & 17.4 & 20.0 & 21.8 & 24.5 & 27.0 & - \\
& $\varepsilon_{\infty}$ & 1.89 & 1.92 & 1.95 & 1.99 & 2.02 & 2.05 & - \\
\hline \multirow{2}{*}{$\mathrm{C}_{5} \mathrm{H}_{11} \mathrm{OH}$} & $\varepsilon_{0}$ & 12.2 & 14.1 & 16.0 & 17.5 & 19.7 & 21.8 & - \\
& $\varepsilon_{\infty}$ & 1.92 & 1.95 & 1.98 & 2.02 & 2.05 & 2.08 & - \\
\hline
\end{tabular}

1) Debye, Handbuch der Radiologie 6 (1925). 